\title{
Activismo frente a norma: ¿quién salva a la universidad?
}

\author{
Vicente Manzano-Arrondo vmanzano@us.es \\ Universidad de Sevilla, España.
}

\section{Resumen}

La fuerte presión que se está ejerciendo actualmente sobre la educación superior dificulta su capacidad para trabajar directamente hacia el bien común desde su carácter de organización basada en el conocimiento. Esta consecuencia afecta a la relación entre la universidad y el tercer sector, en la puesta en práctica de experiencias de aprendizaje servicio, investigación-acción y otros modelos de sinergia. A partir del análisis de las publicaciones y de entrevistas a cargos académicos y profesorado activista, este documento describe dos perfiles complejos que ayudan a entender las reacciones de los miembros de la academia cuando se enfrentan a estas presiones. Estas reacciones muestran cuatro criterios dicotómicos: actitudes frente a la hegemonía (tendencia de mercado o tendencia social), reacciones a la presión (obediencia o resistencia), imparcialidad académica (neutralidad o posicionamiento) y posturas sobre el pragmatismo (realismo o idealismo). Así, el perfil MOR es definido por estilo de (M)ercado, (O)bediencia y (R)ealismo; mientras que el perfile SRIP es definido por tendencia (S)ocial, (R)esistencia, (I)dealismo y (P)osicionamiento. El trabajo describe las consecuencias en el trabajo universidad-tercer sector y propone vías para solucionar estos problemas.

\section{Palabras clave}

Aprendizaje servicio, investigación-acción, educación superior, mercantilización, institucionalización. 


\title{
Activism Against Standard: Who Saves University?
}

\begin{abstract}
The strong pressure that is now, more than ever, being exerted on higher education is abating its capability to work directly towards the common good from the place of a knowledge-based organization. This result affects the relationship between the university and the third sector via the implementation of service learning, action research and other models of synergy. From the analysis of scientific publications and interviews of both academic managers and activist professors, this document describes two complex profiles (MOR and SRIP) which help make sense of the reactions of university members when facing those pressures. These reactions show four dichotomous criteria: attitudes facing hegemony (market trend versus social trend), reactions to pressure (obedience versus resistance), academic impartiality (neutrality versus positioning), and positions on pragmatism (realism versus idealism). Thereby, MOR profile is defined by (M)arket style, (O)bedience, and (R)ealism, whereas SRIP profile is defined by (S)ocial trend, (R)esistance, (I)dealism, and (P)ositioning. This document describes consequences in university-third sector work, and proposes ways to solve these problems.
\end{abstract}

\section{Keywords}

Learning-Service, Action Research, Higher Education, Commodification, Institutionalization. 


\section{Introducción}

Las instituciones de educación superior se encuentran sometidas a intensas reformas, que generan varias crisis de envergadura en su seno (De Wit, 2010; Jarab, 2008; Manzano-Arrondo, 2009, 2011, 2012, en prensa; Rubalcaba, 2003; Shumar, 2004; Sousa, 2006). Uno de los aspectos que más sobresalen es la creciente presión que sufre el mundo académico para centrar su actividad en la publicación dentro de revistas indexadas en sistemas como el Journal Citation Index (Fernández-Quijada, Masip y Bergillos, 2013; Mateo, 2012; Oyarzun, 2008; Urcelay y Galetto, 2011). Esta actividad focaliza la academia hacia la salvación individual por medio de una continua adaptación, que reduce las posibilidades de modelos de trabajo centrados directamente en la promoción de cambio social desde praxis universidad-calle (en adelante, PUC), como ocurre con los modelos de aprendizaje servicio, investigaciónacción, co-investigación, o unidades de acción comprometida. Así, Fox (2008) pregunta, en otras palabras, ¿cómo implicarse en PUC, cuando las trayectorias académicas recorren caminos más ágiles y desconectados de los problemas reales de la mayoría de la población?

Abordar PUC desde la universidad requiere dosis de activismo y niveles de autonomía, que se ven dificultados por las presiones normativas. Al respecto, la tesis de este trabajo es doble: 1) coexisten dos perfiles de reacción frente a las reformas: seguimiento de la norma frente a activismo, y 2) el perfil que actualmente está guiando el rumbo de las instituciones de educación superior es el seguimiento de la norma. En contraste, abundan las voces que reclaman una universidad al servicio directo del bien común desde lo que le es propio: la construcción, difusión y gestión de conocimiento (Ellacuría, 1999; Johnson y Hirt, 2011; Manzano-Arrondo, 2012; Rodrigues, 2007; Siegel, 1984; Watson, 2008).

Desde una perspectiva provocadora, la universidad necesita ser salvada de esta vorágine de adaptación irreflexiva, para desempeñar su función constructora de mundos mejores. Si sus miembros se encuentran inmersos en tareas de autosalvación y ajuste normativo, ¿¿uién salva a la universidad?

Este documento no pretende responder de forma literal y concluyente a la pregunta, sino ofrecer materia prima para trabajar en la solución. Para ello, el primer apartado aborda los fundamentos teóricos y experienciales que sostienen la existencia de dos perfiles contrapuestos en las instituciones de educación superior, mientras que el segundo ofrece datos que avalan tal distinción. En el último apartado se ofrecen algunas indicaciones que podrían estimular soluciones concretas. 


\section{Marco teórico}

\section{Barreras reformistas para las PUC}

Especialmente desde los años 80, las instituciones de educación superior se encuentran inmersas en un profundo proceso de transformación como resultado de lo que ha sido denominada hegemonía de la globalización neoliberal (De Wit, 2010; Manzano-Arrondo, 2011; Shumar, 2004; Wang, Ling, Spalding, Odell y Klecka, 2011; Yogev y Michaeli, 2011). De acuerdo con este modelo hegemónico, las instituciones de educación superior han de comprometerse activamente con los valores y las prácticas de la globalización mercantil mediante un paquete de medidas que se impone a lo largo del planeta, tal y como se ha denunciado ya en Australia (Worthington y Higgs, 2011), Chile (Spinoza, 2008), Indonesia (Susanti, 2011), Israel (Yogev y Michaeli, 2011), Nueva Zelanda (Codd, 2005), Dinamarca, Finlandia, Noruega y Suecia (Musial, 2010), Grecia (Venieris y Cohen, 2004), Japón (Yamamoto, 2004), España (Manzano-Arrondo y Andrés, 2007) o Turquía (Kennedy, Senses y Ayan, 2011), además de países de la periferia como China, Fiji, Uganda o Kenia (Johnson y Hirt, 2011). Este paquete de medidas es definido mediante 1) a nivel de docencia, formación de profesionales altamente cualificados, disponibles en tiempo, lugar, y ocupación, capaces de adaptarse con anticipación a los cambios dinámicos de los mercados y de formarse a sí mismos; 2) a nivel de investigación, transferencia de tecnología a las empresas, orientación de objetivos y prácticas de investigación hacia ventajas competitivas, y partenariado con las organizaciones privadas del sector productivo; y 3) a nivel institucional, reducción de la financiación pública, fusión de universidades, desaparición de centros públicos, reducción de plantilla en las instituciones de educación superior públicas, adopción de estilos empresariales de gestión y transformación en espacios de negocio.

Respecto al interés de este documento, los efectos que más dificultan el trabajo de la institución y de sus miembros en PUC pueden ser: desánimo de los académicos (Shields y McGinn, 2011); devaluación de personas e instituciones que no se adaptan a estas presiones (Malecki, 2000); aumento del individualismo y del credencialismo (Shumar, 2004), que casa bien con la evangelización en los valores mercantiles (Manzano-Arrondo, 2009) y con la orientación del trabajo académico hacia las demandas del tejido productivo o financiero (de la Corte, 2004; Plaza, 2003); asunción del mínimo coste en las decisiones y prácticas (Worthington y Higgs, 2011); reducción de acciones posibles, en paralelo con la reducción de fondos (Frew, 2006); mutación de las instituciones de educación superior desde agentes de cambio social a instituciones bajo control (García, 2008), que deriva en una definición externa de la agenda universitaria (Filipakkou y Tapper, 2008); orientación de la actividad universitaria hacia el cumplimiento de estándares de calidad importados del mundo 


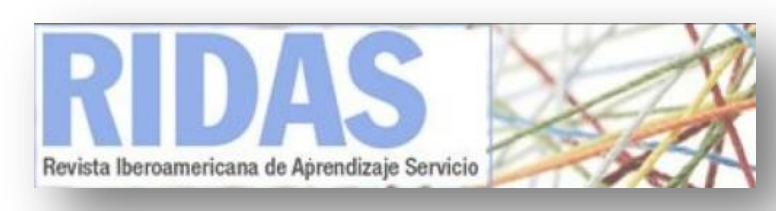

empresarial (Temple, 2005), que mantiene entretenidos a los miembros de la academia en labores de competición (Amigot y Martínez, 2013); fomento de instituciones de educación superior más preocupadas por su imagen que por su función social (Wareass y Solbakk, 2009); imposición de un modelo de universidad desde el Norte hacia el Sur (Rodrigues, 2007), que aumenta el desequilibrio entre ambas partes (Sousa, 2006), e impone un modo de trabajo académico centrado en la competitividad internacional (Spinoza, 2008). Ordine (2013) es muy ilustrativo cuando acusa a las reformas de preocupar y ocupar constantemente al profesorado en tareas que le inhabilitan para lo trascendente. Y Ball (2003) es incisivo cuando presenta a los docentes como seres ontológicamente transformados, tecnificados y perdidos en una lucha interminable por adaptarse a reformas que vacían de sentido su profesión. Como resultado, la técnica sustituye a la ética, y la ejecución al significado.

Se observa en todo ello una notable distancia entre los discursos académicos y los políticos, que se salda a favor de estos últimos (Jarab, 2008; Stensaker, Frolich, Gornitzka y Maasen, 2008; Yamamoto, 2004), plenamente instalados en las características descritas. No obstante, sería deseable que los miembros de la academia adoptaran un papel más propio de instituciones que se consideran sabias y acreedoras de la rebeldía que supone el conocimiento. Ello no implica una postura única. Las instituciones de educación superior no reaccionan de forma unívoca y menos aún sus miembros. Las reformas han sido y son contestadas desde movimientos sociales universitarios, si bien con notables problemas de visibilidad y de capacidad de contagio.

Un análisis en términos de "activismo frente a norma" constituye una simplificación, debido a la complejidad mencionada. Sin embargo, permite suministrar algunas herramientas de intervención. Para entrar en ello, los siguientes epígrafes de este apartado suministran fundamentación sobre dicotomías de acción y reacción, identificables dentro y fuera de la universidad. Estas dicotomías permitirán dibujar dos perfiles más completos.

\section{Fuentes de información}

Este trabajo recurre a fuentes habituales: literatura sobre universidad y prácticas de compromiso; e investigación empírica. Además de ello, la experiencia personal como profesor universitario durante más de veinte años ha sido una base de información que requiere ser descrita.

La experiencia académica pertinente a este trabajo surge principalmente de dos fuentes. Por un lado, se encuentra la membresía en órganos de gestión de la universidad: gestión de departamento y comisiones diversas (calidad, planes de estudio, etc.), además de experiencias normativas (estatutos de la universidad, de la 


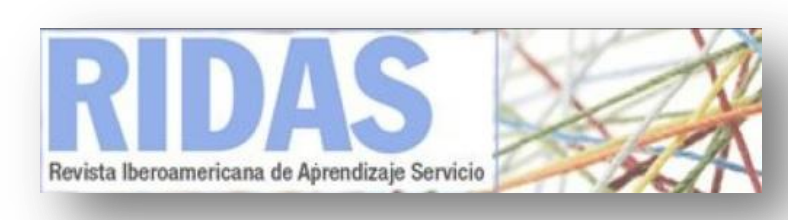

facultad y del departamento). Por otro, coexiste la condición de miembro en diversos colectivos académicos fácilmente calificables como movimientos sociales universitarios interdisciplinares, entre los que se encuentran:

- Otra Investigación es Posible (Díez, Manzano-Arrondo y Torrego, 2013). Orientado a la construcción de relaciones entre: la investigación, las publicaciones y las soluciones concretas de problemas sociales mediante el trabajo conjunto con el tercer sector.

- Universidad y Compromiso Social (Andrés, 2012). Surge con orientación clara hacia dos frentes: promoción de una mirada transdisciplinar de la sociedad local y global, orientada hacia el bien común; y trabajo horizontal con el tercer sector para la solución de problemas concretos.

- Por una Universidad Pública y de Calidad. Su objetivo es la democratización interna de las instituciones de educación superior (con un interés muy especial en los sectores más débiles de la universidad) y la promoción de cambio social.

Estas experiencias personales me han permitido identificar diferentes patrones comunes dentro del quehacer universitario, patrones que son reforzados por los trabajos publicados en la literatura sobre estos asuntos.

Básicamente, estos patrones pueden ser descritos a lo largo de cuatro pares contrapuestos, analizados como las dos caras de cuatro monedas específicas.

\section{Moneda 1: posturas ante la hegemonía}

Las reformas en el sector público se implementan desde la década de los 80 a partir del modelo de la Nueva Gestión Pública, que imita los modos empresariales de gestión, fomentando la competitividad, la privatización y la orientación hacia el cliente (Christensen, 2011). Cuando este modelo aterriza en las instituciones de educación superior genera un discurso de performatividad (Quinn, 2012), caracterizado por un desgaste continuo de la academia a través de la prioridad por resultados medibles, estrategias de control y desprofesionalización (Ball, 2003). Uno de los resultados ha sido la mayor visibilidad de los conflictos (Venieris y Cohen, 2004), especialmente entre los valores académicos y los mercantiles (Moosmayer, 2011). Esta visibilidad ha propiciado que se pronuncien las diferencias entre formas distintas de entender las instituciones de educación superior.

Manzano y Torrego (2009) distinguen tres modelos de trabajo y concepción que coexisten en la universidad. El primero dibuja una institución centrada en el trabajo intelectual y el progreso de la ciencia como resultado del amor por el conocimiento, donde el mundo académico goza de una libertad prioritaria y se autocontrola. El 


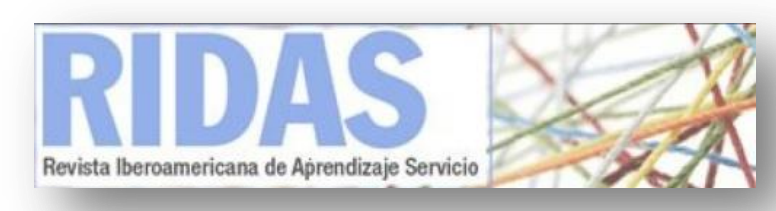

segundo, que a los efectos de este trabajo podemos denominar de orientación mercantil, defiende las bondades del mundo de los negocios; la formación profesional técnica; la implementación de los modelos de calidad, gestión y productividad desde la experiencia empresarial; y la competición entre individuos e instituciones. El tercero, denominado aquí tendencia social, insta a orientar la actividad universitaria hacia la solución de los problemas sociales y medioambientales más urgentes, mediante una profundización en la democracia interna de la institución y la puesta en juego de sus mecanismos de construcción de conocimiento para un trabajo colaborativo con el tercer sector.

Las tendencias mercantil y social constituyen las dos caras de esta primera moneda, asumiendo, cada una a su modo, la faceta académica o intelectual. Wang, Ling, Spalding y Klecka (2011) acuden a la expresión perspectiva de imperativo económico para la primera tendencia, y a perspectiva de resistencia crítica para la segunda. En términos más generales, Morin, Roger y Domingo (2001) utilizan respectivamente las denominaciones hélice cuatrimotor (ciencia-técnica-industria-economía) y hélice humanizadora.

\section{Moneda 2: reacciones frente a la presión}

Individuos y grupos pueden reaccionar de formas muy diferentes cuando se enfrentan a situaciones de presión, opresión o injusticia. Estas reacciones suelen observarse dentro de un continuo que Bacal (1994) sistematiza, acotando tres posturas: conformismo, resistencia y rebelión. En el conformismo, las condiciones no solo son aceptadas, sino que se colabora con el orden establecido. En la resistencia, se mantiene la situación, sin asumir las condiciones de opresión. En la rebelión, se articulan actuaciones concretas orientadas a luchar contra la injusticia. Para Seidman (1988), son también tres las posturas identificables en el continuo: 1) sintonización, acomodación o adaptación, manteniendo el estatus quo; 2) cambio incremental, es decir, lucha o trabajo por lograr un cambio en la línea de mejorar la situación; y 3) reestructuración, como alteración fundamental de las regularidades sociales. Watts, Williams y Jagers (2003) organizan el continuo en cinco etapas, que tienen como punto de mira una alta implicación en la mejora de la sociedad: 1) acrítica: el orden social es interiorizado y se acepta que se vive en un mundo justo; 2) adaptativa: hay conciencia de injusticia, pero se asume como inevitable; 3) precrítica: se cuestiona la propia posición y papel en los procesos y acontecimientos; 4) crítica: hay interés por aprender acerca del funcionamiento de la injusticia y de defender el cambio social; y 5) de liberación: existe un profundo compromiso por la transformación social.

Es posible combinar algunas perspectivas sobre este asunto en un sistema de dos dimensiones, superando la visión del continuo. El cuadro 1 propone una concreción 
basada en dos variables relevantes: 1) la presión que se recibe es asumida literalmente o, por el contrario, transformada; y 2) se ofrece o no resistencia u oposición a cumplir con la presión.

Cuadro 1. Cuatro reacciones frente a la presión. Fuente: elaboración propia

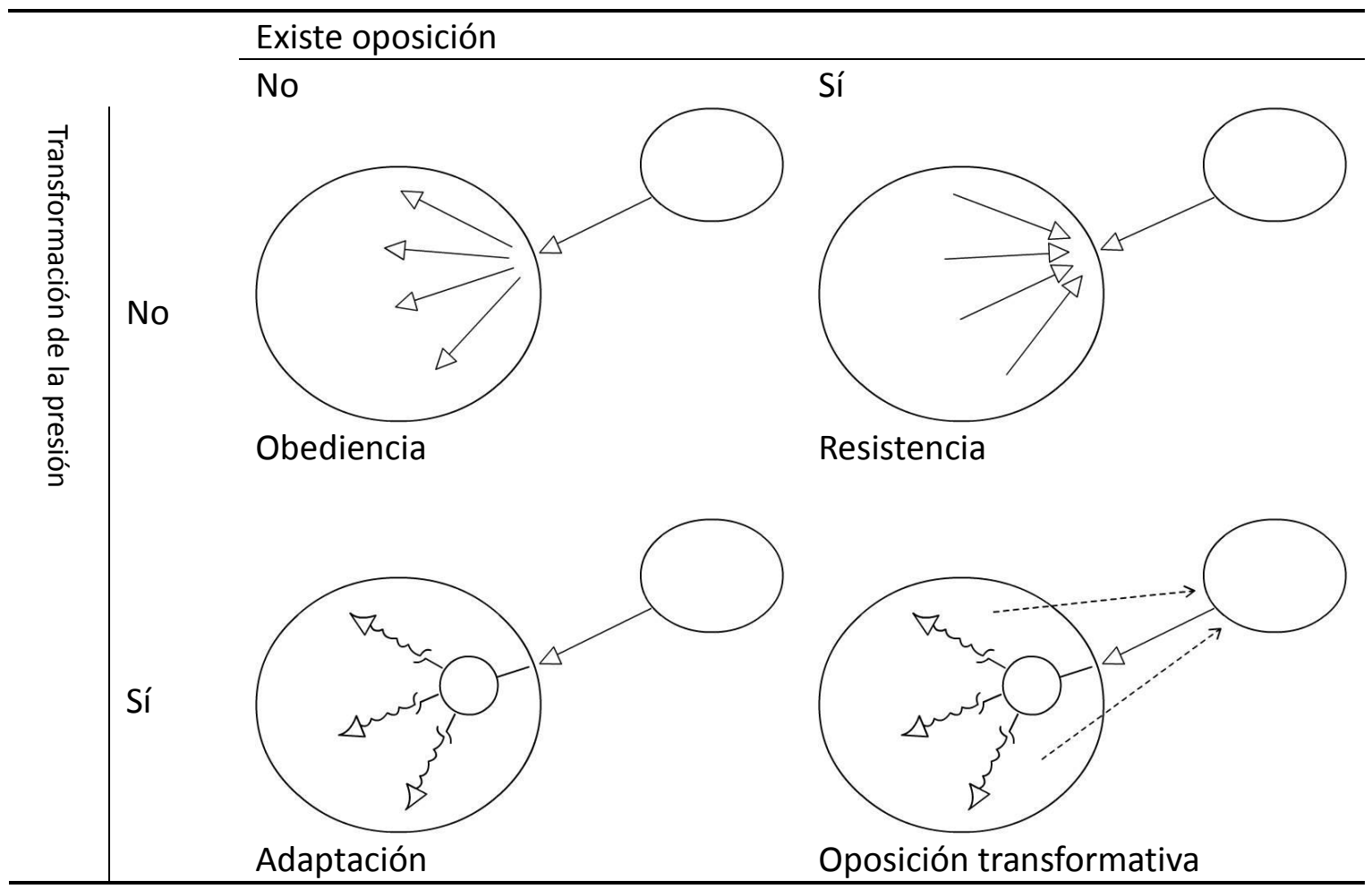

En la cotidianidad es difícil observar modelos puros. Mi experiencia al respecto es que la transformación de la presión resulta más bien anecdótica en las reacciones dentro de la institución, por lo que la práctica descubre especialmente reacciones en torno a la obediencia o a la resistencia. Ambas constituyen las dos caras de esta moneda.

\section{Moneda 3: visiones sobre la imparcialidad académica}

Cuando se pretende indicar que la academia no ha de tomar partido y mantener distancia respecto a los asuntos que se encuentran fuera de sus muros, es habitual confundir los conceptos de objetividad, imparcialidad, neutralidad, distancia y asepsia (Manzano-Arrondo, 2014). Una de las caras de esta moneda puede denominarse defensa de la neutralidad, descrita como la exigencia de que el conocimiento científico que no ha de posicionarse frente a nada que no sea sí mismo, buscando distancia de los acontecimientos y objetividad en el método. Esta pretensión forma parte de la tradición universitaria y de los sistemas educativos en general (Yogev y Michaeli, 


\section{RDAS}

2011). No obstante, se ha denunciado como ilusoria (Barnhizer, 1993) y sorprendente (Ibañez-Martín, 1998). En contraposición, la otra cara de la moneda, defensa del posicionamiento, ha sido descrita como la orientación del saber académico hacia la superación de las estructuras sociales injustas (Ellacuría, 1999), la construcción de justicia social (McArthur, 2011; Rizvi, 2006), el desarrollo de conciencia social, tolerancia y pensamiento crítico (Kennedy, Senses y Ayan, 2011), la modificación de las desigualdades (Apple, 2011), la promoción de rebelión antes que sumisión (Méndez, 2009), o la actitud de servicio a la comunidad (Borba, 2001).

\section{Moneda 4: actitudes acerca del pragmatismo}

Las dos caras de esta moneda, realismo y pragmatismo, requieren no solo de una descripción, sino que procede también señalar el diferente soporte intelectual que han recibido.

\section{Descripción}

Ante cada reforma, una postura realista es salvar la cotidianidad de la institución, gestionando su dinámica de tal forma que la obediencia a la nueva norma permita no poner en peligro la supervivencia o incluso la posición. Este proceder es llamado por Hoekstra y Korthagen (2011) reflejo orientado a la acción, llevado a cabo por quienes Ochoa (2007) denomina optimistas-reformadores y Ball (2003) aplica al ámbito específicamente académico con la expresión profesor postmodernista. La dinámica consiste en garantizar que la institución seguirá siendo administrativamente viable. No existen alternativas juiciosas y el realismo aconseja centrarse en la victoria de los postulados hegemónicos y aprovechar los medios que se disponen para hacer viable las reformas. En muchos casos, incluso se vive con júbilo la promesa de un mejor funcionamiento institucional gracias a las nuevas normativas.

La visión realista puede ser descrita en tres puntos, aplicados al contexto de las instituciones de educación superior:

1. Existen normativas sobre qué ha de hacer la universidad, así como medios legales, institucionales y económicos para aplicarlas.

2. Las IES han escogido o están escogiendo seguir las nuevas normativas, lo que respalda su aplicación generalizada.

3. Si hacemos lo mismo, contaremos con los mismos medios. Si no es así, nos encontraremos en una soledad inviable.

El realismo transita de los medios a los objetivos - ¿qué podemos hacer con lo que tenemos?-; mientras que el idealismo camina de los objetivos a los medios -¿qué hacemos para conseguirlo?-. La postura idealista es definida por Hoekstra y Kortaghen 


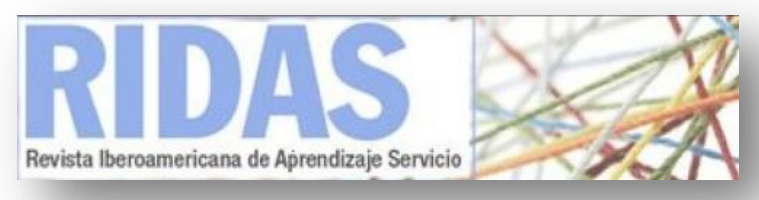

(2011) como reflejo orientado al significado, llevado a efecto por quienes Ochoa (2007) denomina crítico-revolucionarios y, para el ámbito académico, Ball (2003) considera profesor auténtico. En contraposición con la postura pragmática o realista, la visión idealista lleva a primer término elementos humanistas, emancipadores e incluso emocionales. En la práctica, queda definida por tres puntos:

1. Las reglas se establecen para la promoción de bien común. Luego, si no funcionan para tal fin, deben cambiarse.

2. Las IES son organizaciones expertas en crear conocimiento. Luego, si evalúan las nuevas reglas como inoperantes para la construcción de bien común, están capacitadas para proponer otras.

3. Si las IES comienzan a seguir las nuevas reglas, arrastrarán a otras para hacer lo mismo. Siendo conscientes de este resultado, lo peor que pueden hacer es seguir esas reglas, en lugar de denunciar las dinámicas y proponer soluciones.

Soporte intelectual

Existe un amplio elenco de intelectuales que defienden una labor revolucionaria, transformadora y liberadora para las instituciones de educación. Referentes como Jacques Derrida, Paulo Freire, Ignacio Ellacuría, Edgar Morin, Ricardo Petrella o Boaventura de Sousa, entre muchos otros, permiten soporte intelectual para la postura idealista. Esta abundancia no ocurre para la visión realista, pues su argumentación es más pragmática que intelectual. El realismo arranca del principio esto es lo que hay, mientras que el idealismo procede de si no existe, hagámoslo. Esta circunstancia obliga al idealismo a buscar sustento argumentativo, soportes teóricos y justificaciones empíricas que visibilicen alternativas. Por el contrario, el realismo no vive la tensión de justificar algo que ya está funcionando, sea mejor o peor, para bien o para mal.

Para Derrida (2001), la universidad debe ser un punto de resistencia crítica, un espacio inviolable que representa la garantía de la libertad de pensamiento frente a cualquier tipo de poder. Freire (2001) defiende que la alfabetización no es suficiente, que es necesario leer (comprender) y escribir (transformar) el mundo. Insiste en que las personas no son víctimas de la historia, sino sus arquitectos. Luego, postula una educación transformadora que trabaja para la justicia social y la liberación de personas y comunidades. Ellacuría (1999) propone una universidad que trabaja en favor de las personas oprimidas. Critica las instituciones de educación superior que practican revolución desde partidismos políticos o trincheras de calle, tanto como aquellas que abandonan el idealismo y siguen la inercia. La principal característica de la universidad debería ser su apuesta por poner el conocimiento al servicio de la superación de las estructuras sociales injustas. Morin (1995) critica la hiperespecialización académica 
que ha perdido la mirada compleja y por tanto ética sobre el mundo en cuya construcción colabora, e impele a la implicación directa de la educación en la solución de los problemas planetarios reales. Petrella (1997) lleva a cabo una potente defensa del bien común. En ese camino, disecciona las políticas actuales, mostrando su capacidad para destrozar derechos humanos, solidaridad, bienestar y colaboración. La universidad tiene la oportunidad de denunciar estos funcionamientos y de elaborar líneas de trabajo directamente orientadas al bien común. Sousa (2006) muestra las fragilidades de la universidad actual y anuncia desequilibrios futuros. Defiende y define una universidad que trabaja en colaboración con movimientos sociales en contextos multiculturales.

\section{Activismo, norma y perfiles}

A partir de la literatura revisada, es fácil suponer que la conexión entre las opciones dicotómicas combina para formar dos perfiles más o menos nítidos. De este modo, puede hipotetizarse que, en este contexto fuertemente normativizado, los miembros del profesorado que desempeñan cargos de gestión, caracterizan su labor cotidiana desde la tendencia mercantil, la obediencia, la defensa de la neutralidad y el realismo. Por su parte, el profesorado activista, es decir, implicado en PUC y otras tareas similares, transita más bien por la tendencia social, la resistencia, el posicionamiento y el idealismo.

\section{Método}

En la segunda mitad de 2008 se puso en marcha una investigación Delphi, combinada con entrevistas focales, en la que se pretendía averiguar qué piensan los miembros de la universidad sobre la función de su institución. El método Delphi es un procedimiento iterativo donde los expertos conocen los resultados de una ronda antes de participar en la siguiente, desde el anonimato, es decir, desconociendo quién en concreto ha aportado qué cosa (Landeta, 2002). Es un instrumento muy aconsejable en situaciones donde la información que puede manejarse no es precisa y requiere opinión fundamentada (Ruiz e Ispizúa, 1989). Aunque en su origen se utilizaba para realizar previsiones de futuro, hoy en día se lleva a cabo todo un amplio abanico de adaptaciones a situaciones diversas (Callaham, Wears y Weber, 2002). En esta investigación, se acudió como expertos a personal docente e investigador de universidades públicas con suficiente experiencia en algunos de los tópicos abordados: cambios en la normativa universitaria, gestión e implicación con movimientos sociales. Las entrevistas focales, es decir, centradas en el asunto que interesa en cada momento (Pérez, 2010), se utilizaron con algunos miembros con el objetivo de aclarar y profundizar en algunas de sus respuestas. 


\section{Participantes y fases}

Durante la primera fase, participaron 22 miembros del profesorado universitario. Nueve de ellos (grupo GA) contaban con cargos de responsabilidad en la gestión universitaria en general y en la implementación de las reformas derivadas del Espacio Europeo de Educación Superior, en particular. Los 13 participantes restantes (grupo GB) fueron reclutados de una organización estatal de activistas universitarios (Otra Investigación es Posible). En todos los casos, los participantes contaban con el título de doctorado. Respondieron inicialmente a un cuestionario semi-estructurado gestionado mediante correo electrónico.

Las respuestas sirvieron para redactar un informe de síntesis. Se citó a los participantes a una segunda fase, esta vez a través de una página web específica, con el objetivo de garantizar la máxima comodidad y versatilidad. Esta experiencia culminó también con un cuestionario semi-estructurado. El número de participantes decreció para esta segunda fase: 4 para GA y 9 para GB. Esta tasa de abandono es habitual en las encuestas a distancia (Díaz de Rada, 2000). Así mismo, al aplicar una prueba de relación entre el grupo de abandono y tasa, se obtuvo una $\mathrm{V}$ de Cramer de efecto prácticamente nulo ( $\mathrm{V}=.101 ; \mathrm{p}=.549)$. Luego, no podemos suponer covariación entre grupo y abandono, por lo que los resultados no están influenciados por esta dinámica.

Debido al alto nivel de acuerdo en la segunda fase, no fue necesario aplicar una tercera. No obstante, los resultados Delphi fueron completados mediante entrevistas focales con los participantes, con el objetivo de dar pie a una expresión más libre de su posicionamiento, una vez vivida la experiencia de la investigación.

\section{Instrumentos}

La herramienta fundamental para la recogida de datos fue el cuestionario semiestructurado de la primera fase, con las siguientes cuestiones:

- ¿Qué habría que cumplir para formar parte de la comunidad académica? Los participares asumieron el rol de miembros de un tribunal que debía seleccionar a candidatos para la comunidad académica. Los cuatro criterios suministrados fueron: habilidades intelectuales, habilidades sociales, compromiso social y esfuerzo personal. Cada participante contaba con la posibilidad de proponer un quinto criterio. Los cinco se aplicaban a tres situaciones: selección de un estudiante, de un investigador y de un docente.

- ¿Quién debe gobernar la universidad? Los participantes desempeñaron el papel de político legislador. Su función fue ponderar la importancia de doce posibles integrantes del gobierno universitario, cada uno de los cuales era pesado según 
tres criterios: docencia, investigación y administración interna. Los doce posibles integrantes fueron: estudiante, personal de administración y servicios, académico, empresa, sindicato, movimiento social, asociación profesional, partido político, gobierno local, gobierno regional, gobierno estatal y otros.

- ¿Cuáles son los tres problemas más urgentes a los que se enfrenta la sociedad? (Pregunta de respuesta abierta).

- ¿Qué puede hacer la universidad para colaborar en la solución de esos problemas? Grado en que la universidad puede colaborar desde cuatro actividades: educación integral, formación profesional, investigación, e intervención directa.

- ¿Cuáles son los tres principales problemas a los que se enfrenta la universidad para los próximos años? (Pregunta de respuesta abierta).

- Atendiendo a lo que usted considera una universidad ideal (la universidad que debería ser), ¿cuáles son las tres principales oportunidades que se presentan en los próximo años? (Pregunta de respuesta abierta).

- ¿Y las tres principales barreras? (Pregunta de respuesta abierta).

\section{Resultados}

El cuadro 2 muestra una síntesis de los resultados obtenidos durante la primera fase Delphi. Si bien los grupos GA y GB muestran algunas coincidencias importantes, lo más relevante de sus respuestas se encuentra en las diferencias.

Las referencias a la esfera individual frente a la social son más evidentes en GA, mientras que lo contrario es característico de GB. GA enfatiza el mérito para formar parte de la universidad, mientras que GB destaca el compromiso y el origen social. GA anuncia amenazas para la seguridad de los individuos, mientras que GB deplora el decremento de la participación, el humanismo y la actitud crítica.

La posición frente a los valores de mercado es muy diferente en ambos grupos. GA deplora la excesiva regulación estatal, espera mejor preparación de los estudiantes para la competitividad y desea que las empresas adquieran una función importante en la definición de las investigaciones. GB denuncia el papel excesivo de los mercados y la economía en la definición de la universidad, desea una mayor implicación de los movimientos sociales en la universidad (especialmente en el campo de la investigación) y de la universidad en los problemas sociales. 
Cuadro 2.Respuestas al cuestionario de la primera fase. Fuente: elaboración propia

\begin{tabular}{|c|c|c|c|}
\hline \multirow{2}{*}{ Categoría } & \multicolumn{3}{|c|}{ Grupos } \\
\hline & Ambos & $\mathrm{GA}$ & GB \\
\hline $\begin{array}{l}\text { Qué hay que tener } \\
\text { para ser miembro de } \\
\text { la universidad }\end{array}$ & Vocación. & $\begin{array}{l}\text { Esfuerzo. } \\
\text { Habilidades intelectuales. }\end{array}$ & $\begin{array}{l}\text { Compromiso social. Origen } \\
\text { no privilegiado para } \\
\text { estudiantes, habilidades } \\
\text { intelectuales para } \\
\text { investigadores y } \\
\text { habilidades sociales para } \\
\text { docentes. }\end{array}$ \\
\hline $\begin{array}{l}\text { Quién ha de } \\
\text { gobernar la } \\
\text { universidad }\end{array}$ & $\begin{array}{l}\text { Los académicos, con } \\
\text { estudiantes en los } \\
\text { asuntos docentes, }\end{array}$ & $\begin{array}{l}\text { con empresas en } \\
\text { investigación y con } \\
\text { administrativos en } \\
\text { administración. }\end{array}$ & $\begin{array}{l}\text { con movimientos sociales } \\
\text { en investigación, y } \\
\text { administrativos, sindicatos } \\
\text { y estudiantes en } \\
\text { administración. }\end{array}$ \\
\hline $\begin{array}{l}\text { Cuáles son los } \\
\text { principales } \\
\text { problemas de la } \\
\text { sociedad }\end{array}$ & $\begin{array}{l}\text { Daño } \\
\text { medioambiental y } \\
\text { desigualdades. }\end{array}$ & $\begin{array}{l}\text { Amenazas a la seguridad } \\
\text { de los individuos. }\end{array}$ & $\begin{array}{l}\text { Decremento del } \\
\text { humanismo, la } \\
\text { participación pública y la } \\
\text { actitud crítica. }\end{array}$ \\
\hline $\begin{array}{l}\text { Qué puede hacer la } \\
\text { universidad }\end{array}$ & & $\begin{array}{l}\text { Directamente muy poco. } \\
\text { Indirectamente algo, } \\
\text { mediante la preparación } \\
\text { de profesionales. }\end{array}$ & $\begin{array}{l}\text { Directamente no mucho. } \\
\text { Indirectamente mucho } \\
\text { mediante la educación } \\
\text { integral. }\end{array}$ \\
\hline $\begin{array}{l}\text { A qué problemas se } \\
\text { enfrentará la } \\
\text { universidad }\end{array}$ & $\begin{array}{l}\text { Cambios normativos } \\
\text { y en estilos de } \\
\text { enseñanza. } \\
\text { Financiación escasa. }\end{array}$ & $\begin{array}{l}\text { Cambios en la instrucción } \\
\text { de estudiantes. Formación } \\
\text { para la competitividad. }\end{array}$ & $\begin{array}{l}\text { Orientación hacia el } \\
\text { mercado. } \\
\text { Deshumanización de la } \\
\text { educación. }\end{array}$ \\
\hline $\begin{array}{l}\text { Qué oportunidades } \\
\text { muestran los } \\
\text { cambios normativos }\end{array}$ & Movilidad. & $\begin{array}{l}\text { Mejora de la calidad de la } \\
\text { enseñanza y de la gestión. }\end{array}$ & $\begin{array}{l}\text { Mejora de las condiciones } \\
\text { de la enseñanza. } \\
\text { Consideración de } \\
\text { aproximaciones más } \\
\text { sociales y complejas. }\end{array}$ \\
\hline Yqué barreras & $\begin{array}{l}\text { Pobre financiación. } \\
\text { Resistencia frente a } \\
\text { las mejoras. }\end{array}$ & $\begin{array}{l}\text { Excesiva regulación estatal. } \\
\text { Reconocimiento } \\
\text { insuficiente de la docencia. }\end{array}$ & $\begin{array}{l}\text { Liderazgo de los criterios } \\
\text { económicos. }\end{array}$ \\
\hline
\end{tabular}

Ambos grupos muestran una actitud muy diferente respecto a lo que la universidad puede hacer para colaborar en la solución de los grandes problemas. El cuadro 2 muestra que, en términos generales, GB considera a la universidad más relevante que GA en este asunto. El cuadro 3 muestra con más detalle la posición de ambos grupos. Obsérvese que GB otorga a la universidad mayor puntuación en las cuatro categorías 
de acción. La mayor distancia se muestra en la intervención directa, donde la relevancia de GB es un $208 \%$ respecto a GA.

Estas interpretaciones formaron parte del informe que leyeron los participantes en la segunda fase. Su nivel de acuerdo con las conclusiones fue muy elevado: mediana de valor 8 en una escala de 1 a 9. Cada participante mostraba su grado de afinidad con la postura representativa de GA o de GB, para cada una de las preguntas del cuestionario. Como cabría esperar, ambos grupos mostraron mayor afinidad con su propio colectivo de pertenencia. No obstante, los miembros de GB llegaron a escoger conclusiones concretas de GA en un $7 \%$ de las ocasiones, mientras que GA mostró un grado de auto-afinidad del $100 \%$. Este resultado puede ser interpretado en términos de una tendencia a la apertura más característica en GB que en GA.

Cuadro 3. Papel de la universidad en la solución de los grandes problemas. Fuente: elaboración propia

$$
\text { Valores en el intervalo }(0,10)
$$

\begin{tabular}{|c|c|c|c|}
\hline Categoría de acción & & GA & GB \\
\hline Educación integral & & 6,85 & 9,62 \\
\hline Formación profesional & & 7,22 & 8,21 \\
\hline Investigación & & 7,08 & 9,10 \\
\hline Intervención directa & & 3,33 & 6,92 \\
\hline & Media & 6,12 & 8,46 \\
\hline
\end{tabular}

Las entrevistas focales iban encaminadas a profundizar en los aspectos que quedaron menos sólidos tras la recogida grupal. El interés de las respuestas en las entrevistas aflora especialmente durante la construcción de perfiles complejos que se aborda en el siguiente apartado. Por este motivo, las porciones de narrativa son expuestas literalmente en ese momento.

\section{Discusión y conclusiones}

\section{Dos perfiles}

\section{Perfiles complejos}

En la revisión de la literatura, se han señalado cuatro criterios dicotómicos, cada uno de ellos presentados como dos caras de una moneda. Si cada moneda puede no aparecer o hacerlo con una $u$ otra cara, son entonces posibles $3^{4}=81$ combinaciones. 
No obstante, tanto la coherencia lógica entre opciones, como las relaciones dibujadas en la literatura y la experiencia directa, sugieren la existencia de una fuerte relación de diferentes combinaciones entre sí. Así, por ejemplo, el individualismo y el materialismo que forman parte de la perspectiva mercantil, se encuentran también relacionadas con la obediencia como un valor en sí mismo (Passini y Morselli, 2010).

En esta línea, los resultados muestran una alta coincidencia entre diferentes opciones de los criterios dicotómicos, conformando básicamente dos perfiles complejos más o menos nítidos, avalados por los dos colectivos o sectores académicos entrevistados. La figura 1 muestra el perfil Mercado-Obediencia-Realismo (MOR), asociado con el grupo Delphi GA; mientras que la figura 2 representa el perfil Social-Resistencia-IdealismoPosicionamiento (SRIP), asociado con el grupo Delphi GB.
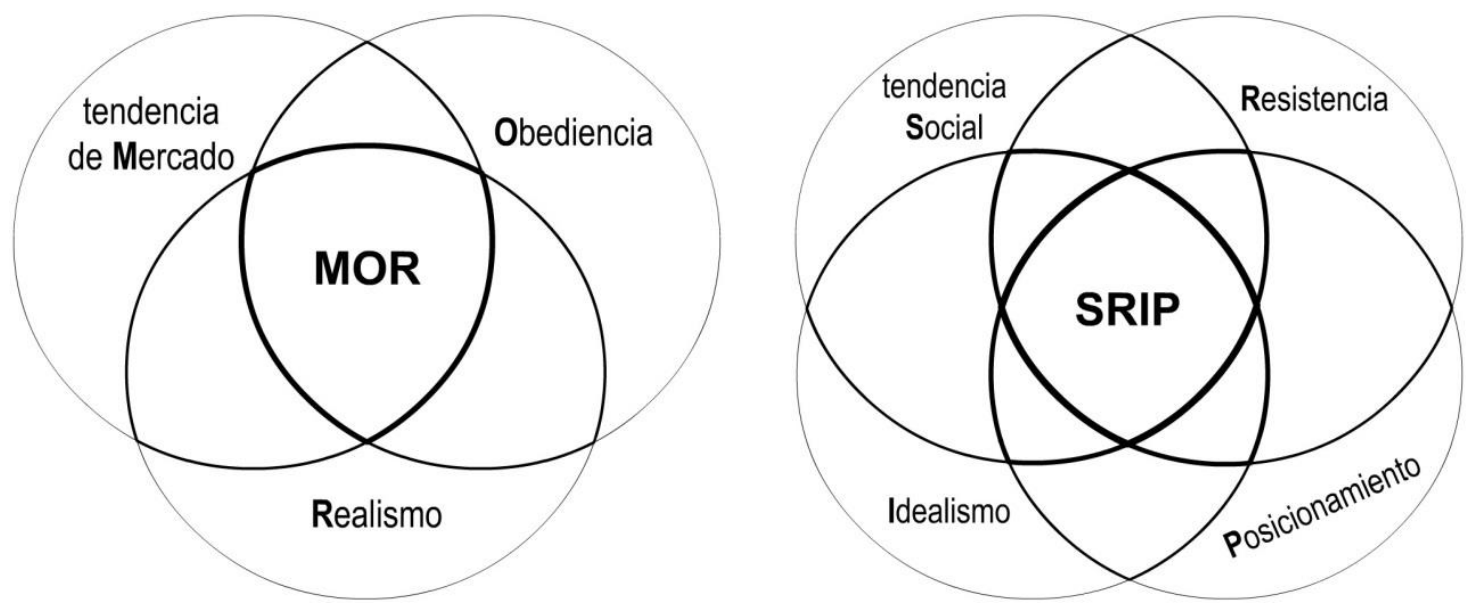

\section{Posturas ante la hegemonía}

MOR adopta la tendencia de mercado al valorar de forma destacada la competitividad y el mérito, apuntando a problemas de naturaleza individual, señalando a las empresas como partenariado importante en la universidad, asumiendo el liderazgo de los modelos de calidad y deplorando la excesiva regulación del estado. SRIP adopta la tendencia social al conceder una importancia visible a los aspectos colectivos, deplorando la pérdida de valores humanos, defendiendo el partenariado con movimientos sociales, la democratización interna de la institución y el origen social de los estudiantes. 


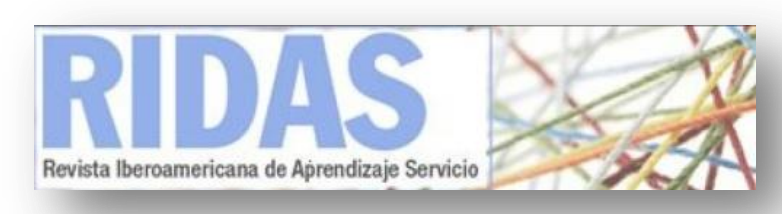

\section{Reacciones frente a la presión}

La obediencia es un asunto incómodo. Es difícil que alguien acepte con agrado ser descrito como obediente, mucho menos en el contexto académico, donde la libertad es un aspecto muy valorado. No obstante, la obediencia es una buena hipótesis parcial para explicar el comportamiento MOR, cuando este perfil se enfrenta a la situación en la que hay que aplicar o no las reformas. No solo las respuestas de los cuadros 2 y 3 responden a una adaptación afín a la norma, sino también las narrativas en las entrevistas. Un miembro GA indicó "Que me guste o no la normativa no es algo importante. Como responsable de la gestión, mi obligación es garantizar que la normativa es aplicada. Es más, debo garantizar la tranquilidad de la universidad para que cuente con normas para todo. Nos guste o no, la ley es garantía". Por el contrario, para SRIP, lo más característico son las referencias a las conductas de resistencia, muy afín también a la fundamentación de movimientos sociales con los que SRIP mantiene sintonía. Algunas porciones de discurso relevantes en dos entrevistas a miembros de GB son "Denuncié que la universidad se había vendido", "No he participado en la implementación de la reforma", "Esto no es más que un paquete de normas que amenazan la esperanza de un mundo mejor desde la universidad. Hemos de pararlo". Este tipo de respuestas, tanto en las expresiones de las preguntas abiertas Delphi como en las entrevistas focales, estuvieron completamente ausentes en GA. Lo más cercano fue la queja más o menos compartida en que las reformas no vienen acompañadas por los medios suficientes para llevarlas a cabo.

\section{Actitudes acerca del pragmatismo}

Durante las entrevistas focales y en algunas respuestas abiertas Delphi, lo más destacado desde GA fue pragmatismo y realismo. Así, un miembro de este grupo respondía "Francamente, no manejo ningún referente teórico ni intelectual para llevar a cabo mi trabajo de gestión. Años de experiencia en la docencia, me han enseñado que para hacer cualquier cosa hay que contar con medios para ello". La preocupación por resolver efectivamente la cotidianidad de la gestión universitaria, deja poco espacio en la práctica para dar carta de viabilidad a otros modos de resolver problemas o de guiar la institución. Un cargo indicaba "como dices, sería reconfortante trabajar con movimientos sociales, pero hay que reconocer que al menos a corto y medio plazo eso es imposible". En contraposición, GB mostraba indicios más emocionales, con apertura a modos alternativos de funcionamiento y fuentes diversas de inspiración. Un profesor de este grupo expresaba "me inspiran procesos de lucha actuales en las universidades chilenas, egipcias, estadounidenses, griegas e inglesas, así como otras luchas pasadas como la de mayo del 68. Desde estas luchas cercanas y más lejanas recibo continuamente textos de compañeros y compañeras analizando la situación". 


\section{Visiones sobre la imparcialidad académica}

No hay referencias a la neutralidad en las respuestas Delphi, ni en las entrevistas posteriores. GA no ha mostrado una postura clara y explícita hacia la neutralidad. Defender que la universidad ha de cumplir con su trabajo y que este trabajo no consiste en solucionar directamente problemas sociales no es defender la neutralidad, aunque la refuerce por omisión. No obstante, los miembros GB han manifestado directa e indirectamente claras posturas hacia el posicionamiento, en el sentido de una orientación clara del trabajo académico hacia la solución de los grandes problemas. EI cuadro 3, ya comentado, es muy ilustrativo al respecto. Para apoyar su opción por el posicionamiento, un miembro GB rescató la famosa frase del dramaturgo español José Bergamín: "Si me hubieran hecho objeto, sería objetivo, pero me hicieron sujeto". Otro miembro de este grupo dijo "necesitamos políticas específicas para guiar el trabajo de la universidad hacia la solución de los problemas que afectan a los más marginados".

\section{Visión de conjunto}

Los cuadros 2 y 3, además de respuestas a las entrevistas, muestran una clara desconexión entre el perfil MOR, propio de los puestos con responsabilidad de gestión, y el perfil SRIP, identificable en el activismo universitario. Un miembro GB decía "Escojo el activismo de los movimientos sociales universitarios porque solo así puedo construir una universidad pública de calidad". Los cuadros 2 y 3 abundan en la distancia entre ambos perfiles.

$\mathrm{Si}$ los gestores universitarios escogen el pragmatismo derivado de la obediencia, mientras que los constructores de caminos alternativos los aplican solo en sus pequeñas esferas de control, no podremos esperar nada nuevo en las instituciones de educación superior. Esta conclusión hace a ambos perfiles igualmente responsables. Ambos sufren una mezcla de tragedia y fatalidad en sus conductas cotidianas. MOR cuenta con modelos operativos para gestionar la institución. Estos modelos, gracias a su soporte político y económico, son relativamente sencillos de aplicar. Dejar este camino trazado no es fácil si no se cuenta con alternativas desarrolladas o concretadas a nivel de gestión cotidiana. Por su parte, SRIP se limita a la esfera de pequeña envergadura que controla (prácticas concretas, movimientos concretos), que requieren pocos recursos y se basan en la autonomía académica todavía existente. Mannarini y Fedi (2012) encuentran que el compromiso requiere algún soporte social, cuya existencia es inversamente proporcional al estrés percibido. SRIP cuenta con este soporte, pues trabaja en grupos de resistencia y acción, en los que encuentra semejantes, seguridad y apoyo. Por su parte, MOR encuentra esa seguridad a través del isomorfismo con otras instituciones y de la conformidad con las normas institucionales (Teelken, 2012). 
Sin embargo, la conexión entre MOR y SRIP en la reorientación de las instituciones de educación superior, resulta ideológicamente posible. Dado que MOR no defiende expresamente la neutralidad, el posicionamiento de SRIP no ha de ser un motivo de conflicto. Por otro lado, la obediencia y el realismo MOR están asentados en la seguridad de los caminos compartidos. La sintetización de las experiencias SRIP podría inspirar otros modelos de gestión realistas, en el sentido de que se demuestran viables en la práctica. El único punto descubierto para posibles conflictos es la orientación hacia el mercado o hacia los problemas sociales. Mi impresión, a la luz de los resultados y de la experiencia personal, es que el bien común es un objetivo compartido por ambos perfiles, como se observa en el cuadro 2 respecto a los problemas medioambientales y las desigualdades sociales. La diferencia es más una cuestión de método. MOR deposita su confianza en la normativa y en los funcionamientos mercantiles como procedimientos que generarán bien común, mientras que SRIP prefiere no mediatizarlo y trabajar directamente por ello.

Algunos autores han apuntado posibilidades para el estímulo del compromiso social universitario a nivel institucional, lo que implicaría una transformación del imaginario MOR, en términos de la constancia de viabilidad. Una de estas posibilidades es facilitar experiencias de trabajo conjunto entre universidad y tercer sector donde participe MOR, mostrando que la universidad puede afectar agendas políticas y la vida de las personas en los problemas más urgentes (Strier, 2011). Otra es promover la imagen de instituciones que son responsables frente a la mejora de la sociedad (Warnick y Silverman, 2011). Otra más es adquirir compromisos con comunidades concretas, de tal forma que resulten atractivos desde un punto de vista académico, a la vez que se estimule el asociacionismo, la mentoría de profesorado y la implicación en programas de calidad (Borba, 2001). MacFarlane (2005) apunta como solución, organizar el tiempo y los espacios de la academia de tal forma que den oportunidades al azar, a las tormentas de ideas y a la reflexión, pues la falta de tiempo se relaciona con la falta de compromiso.

En cualquier caso, los desacuerdos no solo se basan en posiciones ideológicas diferentes, sino también en experiencias previas de desacuerdo (Gray, 2004). La búsqueda activa de experiencias positivas de relación entre MOR y SRIP en algún frente común, puede romper ese ciclo y alimentar la construcción de modelos alternativos de gestión, más proclives a la construcción directa de bien común y, por tanto, afines a las PUC.

\section{Qué hacer con las PUC}

Las PUC, como ocurre con cualquier otro proceso humano, pueden considerarse una combinación de fondo y forma. El fondo se refiere a los objetivos últimos, a las 
intenciones que bañan todo el proceso y a la coherencia que los pasos y las decisiones guardan con ese objetivo. En este sentido, se ha explicitado con claridad que el motivo último de las PUC es la transformación social mediante un trabajo horizontal con el tercer sector. Así se ha indicado en aprendizaje servicio (Speck, 2001), investigaciónacción participativa (Brydon-Miller, Greewood y Maguire, 2003), co-investigación (Borio, Pozzi, y Roggero, 2004) y unidades de acción comprometida (Manzano-Arrondo y Suárez, en prensa).

La forma es una dimensión altamente modificable e instrumental, a la que exigimos eficacia y coherencia. En muchas ocasiones, se requiere un claro apoyo institucional. En aprendizaje servicio, por ejemplo, se defiende la complicidad de la administración institucional (Coles, 2005) y las ventajas de las conexiones inter-institucionales (Butin, 2006). No obstante, este apoyo no está exento de peligro, a la luz de las conclusiones de este estudio, pues puede priorizar la forma sobre el fondo.

Aguilera (2005) denomina "función ceremonial" a las prácticas institucionales centradas en el propio mantenimiento de la organización, en ocasiones contrapuestas a la misión de la institución. En esta línea, es frecuente denunciar que tanto los procesos de institucionalización o normativización, como los de mercantilización, se pierden en funciones ceremoniales (Apple, 2011; Jarab, 2008; Manzano-Arrondo, 2012; Ochoa, 2007), domesticando la forma y, con ello, debilitando el fondo; incluso perdiendo la vista a este al centrar los esfuerzos en aquella. Como ya se ha indicado con otras palabras en las primeras secciones de este documento, las regulaciones universitarias están fundiendo estos procesos ceremoniales al institucionalizar la mercantilización. La confluencia de ambas fuerzas resalta la importancia de la función ceremonial. Así, por ejemplo, Wareaas y Solbakk (2009) describen la fuerte tendencia que las instituciones de educación superior están mostrando por centrarse en el diseño y la práctica de la imagen de marca, consumiendo recursos institucionales y adquiriendo un protagonismo creciente al que denominan branding universitario. A esta inclinación se le suma también creciente tendencia a la tecnificación, es decir, al establecimiento de estándares, guías, protocolos y especializaciones que no solo burocratizan hasta la desesperación la cotidianidad universitaria (Ordine, 2013), sino que penetran en los significados, oscureciendo los objetivos últimos y llegando al extremo de tecnificar la ética (Kisnerman, 2001).

Las conclusiones de este trabajo señalan que la gestión de las instituciones de educación superior se lleva a cabo a través de un perfil, el MOR, que deposita en la norma la confianza del funcionamiento institucional. La consideración en paralelo de todas estas circunstancias y funcionamientos, aconseja tener presente el peligro de la función ceremonial y la apuesta decisiva por el fondo de las PUC. A mi entender, esta consciencia requiere: 


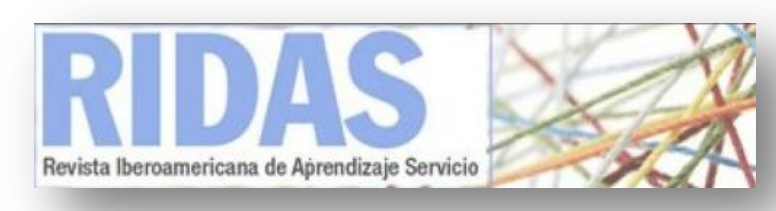

1. Centrar los esfuerzos en una tarea lenta pero imprescindible: la promoción de una cultura universitaria ética, que tiene como una de sus principales consecuencias naturales la apuesta por PUC.

2. Potenciar la implicación de los activistas SRIP en las tareas de gestión de alto nivel y en el asesoramiento del ritmo y orientación institucionales.

3. Propiciar experiencias de relaciones directas con el tercer sector por parte de los cargos MOR. Ello implica, a su vez, aprovechar las oportunidades para que la institución se imbrique en proyectos locales que faciliten el contacto directo, así como abundar en declaraciones o posicionamientos institucionales que visibilicen la posibilidad del compromiso directo ante los propios miembros de la institución y la sociedad local.

4. Utilizar PUC concretas y fácilmente acotadas como vehículo natural para contagiar más experiencias.

5. Fomentar la presencia y visibilidad del tercer sector en la cotidianidad universitaria, mediante su participación en las aulas, en la organización de eventos, en la organización de convocatorias institucionales, en proyectos de investigación, etc.

Estas vías se alimentan mutuamente. Las experiencias de éxito han de ser visibilizadas y contagiar tanto a la base universitaria como a la gestión de alto nivel. Estos referentes pueden ser las herramientas de trabajo para extender una cultura de implicación universitaria hacia el bien común, salvando a la universidad de un destino que tiende a desatender los sectores sistemáticamente más marginalizados de la sociedad.

\section{Referencias bibliográficas}

Aguilera, F. (2005). Los mercados de agua en Tenerife. Bilbao: Bakeaz.

Amigot, P. \& Martínez, L. (2013). Gubernamentalidad neoliberal, subjetividad y transformación de la universidad. La evaluación del profesorado como técnica de normalización. Athenea Digital, 13(1), 99-120. Recuperado de http://psicologiasocial.uab.es/athenea/index.php/atheneaDigital/article/view/1046Amigot.

Andrés, L. (2012). Universidad y Compromiso Social, una experiencia transformadora. En G. Celorio \& A. López de Munain (Coor.), La educación para el desarrollo en la universidad. Reflexiones en torno a una práctica transformadora (pp. 14-22). Bilbao: Hegoa.

Apple, M.W. (2011). Global crises, social justice, and teacher education. Journal of Teacher Education, 62, 222-234. 
Bacal, A. (1994). Types of ethnic identity responses to ethnic discrimination. An experimental approach to Mexican American identity. Göteborgs: Göteborgs Universitet.

Ball, St.J. (2003). Profesionalismo, gerencialismo y performatividad. Revista Educación y Pedagogía, 15(37), 87-104.

Barnhizer, D. (1993). Freedom to do What? Institutional neutrality, academic freedom, and academic responsibility. Journal of Legal Education, 43, 346-357.

Borba, J. (2001). Reducing undergraduate remediation needs through increased university faculty commitment to community service. College Teaching, 49(2), 42-45.

Borio, G., Pozzi, Fr. \& Roggero, G. (2004). La coinvestigación como acción política. En M. Malo (Eds.), Nociones Comunes (pp. 67-78). Madrid: Traficantes de Sueños.

Brydon-Miller, M., Greewood, D. \& Maguire, P. (2003). Why action research?. Action Research, 1(1), 9-28.

Butin, D.W. (2006). The limits of service-learning in Higher Education. The Review of Higher Education, 29, 473-498.

Callaham, M., Wears, R.L., \& Weber, E. (2002). Journal prestige, publication bias, and other characteristics associated with citation of published studies in peer-reviewed journals. Journal of American Medical Association, 287(21), 2847-2850.

Christensen, T. (2011). University governance reforms: potential problems of more autonomy?. Higher Education, 62, 503-517.

Codd, J. (2005). Teachers as 'managed professionals' in the global education industry: the New Zealand experience. Educational Review, 57, 193-206.

Coles, E.A. (2005). Why do Service-Learning? Issues for first-time faculty. En M. Bellner \& J. Pomery (Eds.), Service-Learning: Intercommunity \& Interdisciplinary Explorations (pp. 85-95). Indianopolis: University of Indianopolis Press.

De la Corte, L. (2004). Apuntes sobre crítica y ciencia social: a vueltas con la cuestión del compromiso (I). Realidad, 99, 261-297.

De Wit, K. (2010). The networked university: the structure, culture, and policy of universities in a changing environment. Tertiary Education and Management, 16, 1-14. 


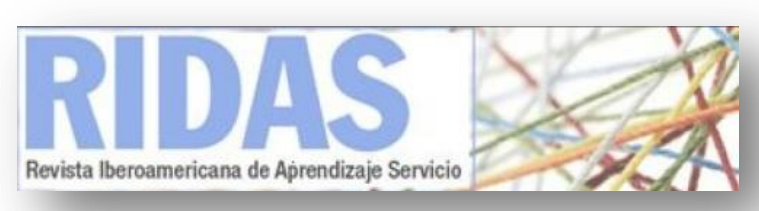

Derrida, J. (2001). Universidad sin condición. Madrid: Trotta.

Díaz de Rada, V. (2000). Problemas originados por la no respuesta en investigación social: definición, control y tratamiento. Pamplona: Universidad Pública de Navarra.

Díez, E.J., Manzano-Arrondo, V. \& Torrego, L. (2013). Otra investigación es posible, una red para tejer sueños. Revista Electrónica Interuniversitaria de Formación del Profesorado, 16(3), 1-10.

Ellacuría, I. (1999). Escritos Universitarios. San Salvador: UCA Editores.

Fernández-Quijada, D., Masip, P. \& Bergillos, I. (2013). El precio de la internacionalidad: la dualidad en los patrones de publicación de los investigadores españoles en comunicación. Revista Española de Documentación Científica, 36(2). Recuperado de http://dx.doi.org/10.3989/redc.2013.2.936.

Filippakou, O. \& Tapper, T. (2008). Quality assurance and quality enhancement in higher education: Contested territories? Higher Education Quartely, 62, 84-100.

Fox, D. (2008). Confronting psychology's power. Journal of Community Psychology, 36, 232-237.

Freire, P. (2001). Pedagogía de la indignación. Madrid: Morata.

Frew, Ch. (2006). An international educational literacy: Students, academics and the state. Journal of University Teaching and Learning Practice, 3, 24-33.

García, M.J. (2008). El impacto de la globalización en la universidad del siglo XXI. Tendencias Pedagógicas, 13, 59-78.

Gray, B. (2004). Strong opposition: frame-based resistance to collaboration. Journal of Community \& Applied Psychology, 14, 166-176.

Hoekstra, A. \& Korthagen, F. (2011). Teacher learning in a context of educational change: informal learning versus systematically supported learning. Journal of Teacher Education, 62, 76-92.

Ibañez-Martín, J.A. (1998). Los códigos de ética profesional de los profesores: ¿simple receta o signo de una nueva educación? En Fr. Altarejos, J.A. Ibañez-Martín, J.A. Jordán \& G. Jover (Eds.), Ética Docente (pp. 61-66). Barcelona: Ariel. 
Jarab, J. (2008). Reforming systems and institutions of higher education: Towards the creation of a European and global higher education area. Education, Citizenship and Social Justice, 3, 85-96.

Johnson, A.T. \& Hirt, J.B. (2011). Reshaping academic capitalism to meet development priorities: the case of public universities in Kenya. Higher Education, 61, 483-499.

Kennedy, N.F., Senses, N. \& Ayan, P. (2011). Grasping the social through movies. Teaching Higher Education, 16, 1-14.

Kisnerman, N. (2001). Ética ¿para qué? En N. Kisnerman (Eds.), Ética, ¿discurso o una práctica social? (pp. 107-121). Buenos Aires: Paidós.

Landeta, J. (2002). El método Delphi. Una técnica de previsión del futuro. Barcelona: Ariel.

Macfarlane, $\mathrm{Br}$. (2005). The disengaged academic: the retreat from citizenship. Higher Education Quartely, 59, 296-312.

Mannarini, T. \& Fedi, A. (2012). Persisting or withdrawing? An insight into the psychological processes underlying sustained engagement. Journal of Community \& Applied Social Psychology, 22, 300-315.

Manzano-Arrondo, V. (2009). Qué cosa es esa de la universidad privatizada. Opciones Pedagógicas, 39, 112-124.

Manzano-Arrondo, V. (2011). El papel de la universidad en la sociedad de ignorantes. Cientifica, 12, 29-55.

Manzano-Arrondo, V. (2012). La Universidad Comprometida. Vitoria: Hegoa.

Manzano-Arrondo, V. (2014). II Barómetro Cittadino. La risposta dell'Universitá per unire didattica, ricerca, azione. Rivista Internazionale di EDAFORUM, 9(23). Disponible en http://rivista.edaforum.it/numero23/monografico_Manzano.html

Manzano-Arrondo, V. (en prensa). Academia, evaluación y poder. RASE.

Manzano-Arrondo, V. \& Andrés, L. (2007). El diseño de la nueva universidad europea. Algunas causas, algunas consecuencias. Sevilla: Atrapasueños. 
Manzano-Arrondo, V. \& Suárez, E. (en prensa). Unidad de acción comprometida: una propuesta de solución ante el problema universitario del servicio a la sociedad. Hábitat y Sociedad.

Manzano-Arrondo, V. \& Torrego, L. (2009). Tres modelos para la universidad. Revista de Educación, 350, 477-489.

Mateo, J.LI. (2012). Les revistes d'impacte i el nou ordre científic mundial. L'Avenç, 383, 41-49.

McArthur, J. (2011). Exile, sanctuary and diaspora: mediations between higher education and society. Teaching Higher Education, 16, 579-589.

Méndez, M. (2009). ¿Sociedades de conocimientos o culturas del saber? Teoría y Praxis, 14, 49-63.

Moosmayer, D.C. (2011). Professors as value agents: a typology of management academic's value structures. Higher Education, 62, 49-67.

Morin, E. (1995). Introducción al pensamiento complejo. Barcelona: Gedisa.

Morin, E., Roger, E. \& Domingo, R. (2001). Educar en la era planetaria. El pensamiento complejo como Método de aprendizaje en el error y la incertidumbre humana. Valladolid: Universidad de Valladolid.

Musial, K. (2010). Redefining external stakeholders in Nordic higher education. Tertiary Education and Management, 16, 45-60.

Ochoa, L. (2007). Resistencia o claudicación. Apuntes sobre la labor intelectual en América Latina. Bajo el Volcán, 7(11), 127-152.

Ordine, N. (2013). La utilidad de lo inútil. Manifiesto. Barcelona: Acantilado.

Oyarzun, R. (2008). Ciencia, revistas científicas y el Science Citation Index: o cómo volvernos locos a golpe de números. REDVET. Revista Electrónica de Veterinaria, 9(9), 1-6. Recuperado de http://www.veterinaria.org/revistas/redvet/n090908.html

Passini, S. \& Morselli, D. (2010). The obedience-desobedience dynamic and the role of responsibility. Journal of Community \& Applied Social Psychology, 20, 1-14.

Pérez, F. (2010). La entrevista como técnica de investigación social. Fundamentos teóricos, técnicos y metodológicos. Acontece, 13. 


\section{RDAS}

Petrella, R. (1997). El Bien Común. Elogio de la solidaridad. Madrid: Debate.

Plaza, S. (2003). Tendencias de cara al proceso actual de reforma de la educación universitaria. En J.M. Sanz \& J.M. Gómez (Eds.), Universidad... ¿Para qué? (73-83). Madrid: Universidad de Alcalá.

Quinn, L. (2012). Understanding resistance: an analysis of discourses in academic staff development. Studies in Higher Education, 37, 69-83.

Rizvi, F. (2006). Imagination and the globalization of educational policy research. Globalization, Societies and Education, 4, 193-205.

Rodrigues, M.A. (2007). El regional y el global: la articulación de la diversidad de funciones de la enseñanza superior. Revista de Universidad y Sociedad del Conocimiento, 4, 5-15.

Rubalcaba, L. (2003). Universidad y construcción social: algunas claves desde el contexto socioeconómico. En J.M. Saz Díaz \& J.M. Gómez Pulido (Eds.), Universidad... ¿Para qué? (141-155). Madrid: Universidad de Alcalá.

Ruiz, J.I. \& Ispizúa, M.A. (1989). La descodificación de la vida cotidiana. Métodos de investigación cualitativa. Bilbao: Universidad de Deusto.

Seidman, E. (1988). Back to the future, community psychology: Unfolding a theory of social intervention. American Journal of Community Psychology, 16, 3-24.

Shields, C. \& McGinn, M. (2011). The erosion of academic troth: disengagement and loss. Teaching Higher Education, 16, 471-482.

Shumar, W. (2004). Global pressures, local reactions: higher education and neo-liberal economic policies. International Journal of Qualitative Studies in Education, 17, 823839.

Siegel, B.L. (1984). Knowledge with Commitment: teaching is the central task of the university. Vital Speeches of the Day, 50(13), 394-397.

Sousa, B. (2006). La universidad popular del siglo XXI. Lima: Fondo Editorial de la Facultad de Ciencias Sociales.

Speck, B.W. (2001). Why Service-Learning? New Directions for Higher Education, 114, 3-13. 


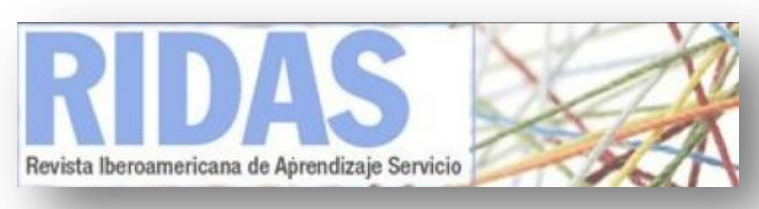

Spinoza, O. (2008). Creating (in) equalities in access to higher education in the context of structural adjustment and post-adjustment policies: the case of Chile. High Education, 55, 269-284.

Stensaker, Bj., Frolich, N., Gornitzka, A. \& Maasen, P. (2008). Internationalization of higher education: the gap between national policy-making and institutional needs. Globalization, Societies and Education, 6, 1-11.

Strier, R. (2011). The construction of university-community partnerships: entangled perspectives. Higher Education, 62, 81-97.

Susanti, D. (2011). Privatization and marketization of higher education in Indonesia: the challenge for equal access and academic values. Higher Education, 62, 209-218.

Teelken, Ch. (2012). Compliance or pragmatism: how do academics deal with managerialism in higher education? A comparative study in three countries. Studies in Higher Education, 37, 271-290.

Temple, P. (2005). The EFQM excellence model: Higher education's latest management fad?. Higher Education Quartely, 59, 261-274.

Urcelay, C. \& Galetto, L. (2011). ¿Editar o no editar?: reflexiones sobre las revistas científicas regionales y algunas propuestas. Kurtziana, 36(1), 3-7.

Venieris, G. \& Cohen, S. (2004). Accounting reforms in Greek universities: a slow moving process. Financial Accountability \& Management, 20, 183-204.

Wang, J.E., Ling, E., Spalding, S.J., Odell, S.J. \& Klecka, C.L. (2011). Understanding higher education in an era of globalization. Journal of Teacher Education, 62, 115-120.

Wareaas, A. \& Solbakk, M.N. (2009). Defining the essence of a university: lessons from higher education branding. Higher Education, 57, 449-462.

Warnick, B.R. \& Silverman, S.K. (2011). A framework for professional ethics courses in teacher education. Journal of Teacher Education, 62, 273-285.

Watson, D. (2008). The university in the modern word. Ten lessons of civic and community engagement. Education, Citizenship and Social Justice, 3, 43-55.

Watts, R.J., Williams, N.Ch. \& Jagers, R.J. (2003). Sociopolitical Development. American Journal of Community Psychology, 31(1/2), 185-194. 


\section{RDAS}

Worthington, A.C. \& Higgs, H. (2011). Economies of scale scope in Australian higher education. Higher Education, 62, 387-414.

Yamamoto, K. (2004). Corporatization of national universities in Japan: revolution for governance or rhetoric for downsizing? Financial Accountability \& Management, 20, 153-181.

Yogev, E. \& Michaeli, N. (2011). Teachers as society-involved "organic intellectuals": training teachers in a political context. Journal of Teacher Education, 62, 312-324.

Nota final

Este trabajo se ha nutrido del apoyo a diferentes niveles de Luis Torrego (pedagogía crítica), Virginia Paloma (psicología comunitaria), Luis Andrés (economía aplicada) y Hassan Fazeli (psicología experimental). En sentido contrario, la investigación ha sido posible a pesar de las importantes barreras que el gobierno de España está imponiendo para el desarrollo de un trabajo académico laboralmente digno y socioambientalmente comprometido. 